\title{
El canal de préstamos bancarios en el Perú: un enfoque VECMI
}

\section{The bank lending channel in Peru: a VECM approach}

\author{
Paul Christian Espinoza Ipanaque ${ }^{2}$ \\ Instituto de Formación Bancaria IFB Certus \\ pespinozai@certus.edu.pe \\ https://orcid.org/0000-0003-2115-8395
}

\section{RESUMEN}

Se busca determinar la existencia del canal de préstamos bancarios en moneda nacional y extranjera para el Perú durante el periodo que va desde el primer trimestre de 2010 hasta el primer trimestre de 2019. Para lograr tal objetivo, se emplean series de tiempo en modelos de corrección de errores VECM, siguiendo la relación de un modelo teórico IS-LM con sector bancario. Se encuentra evidencia de que el canal de préstamos bancarios en moneda nacional estaría operando. A su vez, los fondos de encaje en moneda extranjera son buenos predictores en el largo plazo del nivel de créditos en moneda extranjera; sin embargo, no existe un canal crediticio en dicha moneda. Esta investigación sirve para esclarecer el mecanismo de transmisión de la política monetaria a través del crédito hacia la actividad económica. Además, es útil para el diseño y aplicación de la política monetaria. Finalmente, este es el primer paper en usar la metodología VECM para estudiar el canal de préstamos bancarios en el Perú.

Palabras Clave: Modelo de corrección de errores; canal de préstamos bancarios; política monetaria.

Clasificación JEL: E51; E52; E58

(C) Los autores. Este artículo es publicado por Pensamiento Crítico de la Facultad de Ciencias Económicas, Universidad Nacional Mayor de San Marcos. Este es un artículo de acceso abierto, distribuido bajo los términos de la licencia Creative Commons Atribucion - No Comercia_Compartir Igual 4.0 Internacional. (http:// creativecommons.org/licenses/by-nc-sa/4.0/) que permite el uso no comercial, distribución y reproducción en cualquier medio, siempre que la obra original sea debidamente citada. 


\section{ABSTRACT}

It seeks to determine the existence of the bank loan channel in national and foreign currency for Peru during the period from the first quarter of 2010 to the first quarter of 2019. To achieve this objective, time series are used in correction models of VECM errors, following the relationship of a theoretical IS-LM model with the banking sector. Evidence is found that the bank loan channel in national currency would be operating. In turn, foreign exchange reserve funds are good long-term predictors of the level of foreign currency credits, however there is no credit channel in that currency. This research serves to clarify the mechanism of transmission of monetary policy through credit towards economic activity. In addition, it is useful for the design and application of monetary policy. Finally, this is the first paper to use the VECM methodology to study the bank loan channel in Peru.

Keywords: Error correction model; bank lending channel; monetary policy.

Jel Classification: E51; E52; E58. 


\section{Introducción}

La crisis financiera internacional (CFI) evidenció la importancia del adecuado comportamiento del sistema financiero en la actividad económica (Lahura y Vargas, 2013) y Bustamante, Cuba y Nivin (2019). Los efectos de la CFI impactaron negativamente sobre el principal instrumento de política monetaria en el Perú; la tasa de interés interbancaria, lo cual afectó al manejo de la política monetaria (Vega y Chávez, 2017). En este contexto, es de vital importancia estudiar los mecanismos de transmisión de política monetaria a través de los créditos bancarios sobre la actividad económica. Por ello, el Banco Central de Reserva del Perú BCRP utiliza diferentes instrumentos de política monetaria, como por ejemplo, el encaje bancario, que complementa a su instrumento de política convencional (Armas, Castillo y Vega, 2014).

El canal de préstamos bancarios propuesto inicialmente por (Bernake y Blinder, 1988), considera que a través de la política monetaria se puede afectar el nivel de fondos de encaje de las entidades bancarias y, a su vez, el nivel de oferta de fondos prestables. Por esto, se podría impactar al nivel de actividad económica, ya que la inversión y el consumo dependen de los créditos bancarios. Cabe señalar que la efectividad de este canal se incrementa cuando los pasivos de los bancos dependan, en gran medida, de los depósitos. En el caso peruano, la profundización financiera, medida con el ratio créditos $/ \mathrm{PBI}^{3}$, en el primer trimestre de 2010 fue de $26 \%$ y en el primer trimestre de 2019 es de 50\%. Es decir, este incremento del volumen de créditos en la economía hace más importante la identificación del canal de préstamos bancarios (Carrera, 2011).

El Perú es una economía emergente pequeña porque sus decisiones económicas no impactan en el resto del mundo, y es abierta porque compra y vende productos y servicios del exterior (Castillo, Montoro y Tuesta, 2006). En este contexto, es sumamente importante considerar los efectos de los choques de política monetaria sobre el nivel de créditos y la actividad económica. Más aún, que el BCRP viene implementando y utilizando una serie de instrumentos no convencionales, por ejemplo, el uso activo del requerimiento de encajes en moneda nacional y extranjera (Armas y otros, 2014), además el Programa de Desdolarización financiera de 2013 que ha tenido impacto sobre el traspaso de créditos en dólares por soles 
en los bancos (Contreras y otros, 2019). Así, conforme avanza el programa, debería contribuir al fortalecimiento del canal de crédito (Rossini y Vega, 2007). Por esta razón, la utilización de series agregadas para establecer posibles choques y relaciones de largo plazo es sumamente relevante para el canal de préstamos bancarios en el Perú.

El presente documento de investigación, se enfoca en determinar la existencia del canal de préstamos bancarios en moneda nacional y extranjera durante el periodo que va desde el primer trimestre de 2010 hasta el primer trimestre de 2019. A continuación, se enmarca las siguientes interrogantes: ¿Existe una relación de largo plazo entre los fondos de encaje en moneda nacional con el nivel de créditos agregados en soles? ¿Existe una relación de largo plazo entre los fondos de encaje en moneda nacional con el PBI real? ¿Existe una relación de largo plazo entre los fondos de encaje en moneda extranjera con el nivel de créditos agregados en dólares? ¿Existe una relación de largo plazo entre los fondos de encaje en moneda extranjera con el PBI real?

Para lograr determinar la existencia del canal de préstamos bancarios, este trabajo de investigación se inspira en la metodología usado por los autores (Su, Gan y Hu, 2010) y Mello y Pisu (2009). Primero, se trata de encontrar la integración entre las series agregadas fondos de encaje en moneda nacional y extranjera, nivel de créditos agregados en moneda nacional y extranjera, tasa activa en moneda nacional y extranjera, tasa de rendimiento de los bonos de Perú y Estados Unidos, y el PBI real. Segundo, a través del método de Johansen (1990) se trata de encontrar una relación de equilibrio a largo plazo entre las variables, tal como lo sugiere (Carrera, 2011). Finalmente, estimar modelos de corrección de erros tipo VECM para estudiar y cuantificar las elasticidades en las variables. Cabe precisar que las relaciones de corto y largo plazo se determinan a través de estos modelos (Nzengue y Faycal, 2011).

De este modo, encontramos los siguientes resultados: (i) existe una relación de largo plazo entre los fondos de encaje en moneda nacional y el nivel de préstamos en soles, lo cual ante un incremento en un punto porcentual de los fondos de encaje en soles causan un aumento de los créditos en soles de 2,22\%; (ii) existe una relación de largo plazo entre los fondos de encaje en moneda nacional y el PBI real, así ante un incremento de 1\% 
de los fondos de encaje en soles se produce un incremento del PBI real de $0,50 \%$ en largo plazo; (iii) los fondos de encaje en moneda extranjera son buenos predictores de los créditos en dólares, de esta manera un incremento de $1 \%$ en los fondos de encaje en moneda extranjera causan un aumento de 4,89\% en los créditos en dólares; $y$, (iv) los fondos de encaje en dólares no son buenos predictores de la actividad económica. En concreto, se determina que el canal de préstamos bancarios en moneda nacional estaría operando durante el periodo de análisis. Sin embargo, no existe evidencia empírica sobre un canal de créditos en moneda extranjera.

A diferencia de (Su, Gan y Hu, 2010; Mello y Pisu, 2009), no vamos a utilizar restricciones en los modelos de corrección de errores VECM para la identificación de la oferta y demanda de créditos, ya que derivamos la relación de las variables agregadas del canal de préstamos bancarios de un modelo IS-LM con sector bancario propuesto por (Bernanke y Blinder, 1988). Cabe precisar que este es el primer estudio en tratar de determinar el canal de préstamos bancarios en el Perú con la metodología VECM, además, el primero en utilizar las series agregadas total de encaje bancario en moneda nacional y extranjera.

Finalmente, esta investigación sirve para esclarecer el mecanismo de transmisión de la política monetaria a través del crédito hacia la actividad económica ${ }^{4}$, ya que la evidencia no es concluyente en el Perú (Viladecut y Cabello, 2014). Además, es útil para el diseño y aplicación de la política monetaria.

\section{Metodología}

\subsection{Estacionariedad}

Un proceso estocástico es una secuencia de números aleatorios, por ejemplo, se tiene $\left\{\mathrm{y}_{\mathrm{i}}\right\}$ para $i=1,2, \ldots$. De este modo, si el subíndice representa al tiempo, ésta será una serie de tiempo. Sin embargo, las series de tiempo representan una posible realización de infinitas posibilidades. En términos ideales, no se puede conocer los parámetros poblacionales. En tal sentido, necesitamos series de tiempo donde sus estadísticos provengan de una misma distribución de probabilidad, cuyo set de observaciones sean consistentes con sus parámetros poblacionales (Court y Williams, 2011). 
En síntesis, una serie $\left\{\mathrm{y}_{\mathrm{i}}\right\}$ para $i=1,2, \ldots T$ es débilmente estacionaria si cumple con lo siguiente:

$$
\begin{aligned}
& E\left(y_{i}\right)=u \\
& E\left(y_{i}-u\right)^{2}=\sigma^{2}<\infty \\
& \operatorname{cov}\left(y_{i}, y_{i-j}\right)=\gamma_{i-(i-j)}=\gamma_{j}
\end{aligned}
$$

La ecuación (1) nos indica que las observaciones de una serie de tiempo tendrán media constante. La ecuación (2) trata sobre la varianza de las observaciones que debe ser constante. Finalmente, en la ecuación (3) el término $y_{j}$ representa la autocovarianza de la serie de tiempo.

\subsection{El método aumentado de Dickey-Fuller (ADF)}

Se tiene el siguiente modelo autorregresivo AR en su forma básica:

$$
y_{t}=\alpha \times y_{t-1}+u_{t}
$$

A su vez, para determinar si el proceso autorregresivo de la ecuación (4) es estacionario, puede ser escrito de la siguiente forma:

$$
(1-\alpha \times P) \times y_{t}=u_{t}
$$

Para determinar si la ecuación (5) es estacionaria, se requiere que todas las raíces de la ecuación sean mayores a uno en valor absoluto (Court y Williams, 2011). Por lo tanto, el polinomio a resolver se puede expresar de la siguiente manera:

$$
1-\alpha \times w=0
$$

Donde las soluciones ${ }^{w}$ se pueden representar con la siguiente ecuación:

$$
w^{*}=\frac{1}{\alpha}
$$

Entonces, para que se cumpla la estacionariedad en la serie de tiempo $y_{t},\left|w^{*}\right|>1$ y $|\alpha|<1$.

De este modo, si se tiene una serie con $|\alpha|=1$, se dirá que presenta raíz unitaria y, por lo tanto, será no estacionaria. 
En línea con Locmelis y Mititel (2015) y, sabiendo que, $(\alpha-1)=\delta^{*}$ se presenta tres versiones del método aumentado de Dickey-Fuller (ADF) para determinar si una serie de tiempo presenta raíz unitaria.

Test de raíz unitaria sin intercepto y sin tendencia.

$$
\Delta y_{t}=\delta^{*} y_{t-1}+\sum_{i=1}^{k} \delta_{i} \Delta y_{t-1}+u_{t}
$$

Test de raíz unitaria con intercepto

$$
\Delta y_{t}=\beta_{0}+\delta^{*} y_{t-1}+\sum_{i=1}^{k} \delta_{i} \Delta y_{t-1}+u_{t}
$$

Test de raíz unitaria con intercepto y tendencia determinística.

$$
\Delta y_{t}=\beta_{0}+\delta^{*} y_{t-1}+\sum_{i=1}^{k} \delta_{i} \Delta y_{t-1}+\beta_{1} t+u_{t}
$$

Donde, $\beta_{0}$ es el intercepto, $t$ es una tendencia lineal y $u_{t}$ es el término de error. En este contexto, la hipótesis a probar son las siguientes:

$$
\begin{aligned}
& \mathrm{H}_{0}: \delta^{*}=0 \\
& \mathrm{H}_{1}: \delta^{*}<0
\end{aligned}
$$

La hipótesis nula determina que la serie presenta raíz unitaria, por lo tanto, sería una serie no estacionaria.

Cabe precisar, que la prueba ADF ha sido utilizada para determinar la estacionariedad de las series en el canal de préstamos bancarios (Caglarirmak y Karahan, 2016; Sun, Gan y Hu, 2010; Bringas y Tuesta, 1997).

\subsection{Causalidad de Granger}

Siguiendo la explicación de Court y Williams (2011), se tiene el siguiente modelo de vectores autorregresivos VAR bivariado: 
$\left|\begin{array}{l}y_{1, t} \\ y_{2, t}\end{array}\right|=\left|\begin{array}{l}\delta_{1} \\ \delta_{2}\end{array}\right|+\left|\begin{array}{ll}\varphi_{11} & \varphi_{12} \\ \varphi_{21} & \varphi_{22}\end{array}\right| \times\left|\begin{array}{l}y_{1, t-1} \\ y_{2, t-1}\end{array}\right|+\left|\begin{array}{ll}\omega_{11} & \omega_{12} \\ \omega_{21} & \omega_{22}\end{array}\right| \times\left|\begin{array}{l}y_{1, t-2} \\ y_{2, t-2}\end{array}\right|+\left|\begin{array}{l}u_{1, t} \\ u_{2, t}\end{array}\right|$

El concepto de causalidad propuesto por Granger (1988), determina si los rezagos de las variables de la ecuación (11) ejercen o causan algún tipo de impacto sobre las variables endógenas del sistema. Cabe señalar que los autores Bringas y Tuesta (1997), indican que el test no debe de usarse como un indicador de causa y efecto, ni de algún tipo de direccionalidad entre las variables. En tal sentido, ellos proponen utilizar el test para ver si una variable puede predecir mejor a otra.

Asimismo, se incluye el test de causalidad de Granger para respaldar los resultados antes de estimar una posible relación de largo plazo entre las series de tiempo (Vega y Chávez, 2017).

\subsection{Modelo vectorial de corrección de errores VECM}

La ecuación (11) representa un modelo VAR con dos variables. Asimismo, podemos observar cómo los rezagos de las variables pueden impactar en todas las variables endógenas del sistema. Cabe precisar que las variables incluidas en un modelo VAR tienen que ser estacionarias, es decir, si se cuentan con variables que presentan una tendencia estocástica, como por ejemplo el PBI, por lo general se tienen que diferenciar para convertirlas en estacionarias. Sin embargo, cuando se modela series en diferencias se asume implícitamente que se está trabajando con relaciones de corto plazo. Por esta razón, no modelamos un VAR, ya que nos interesa determinar la existencia del canal de préstamos bancarios en el largo plazo.

Cuando se cuenta con series de tiempo con tendencia estocástica o que no sean estacionarias, se podría evaluar la existencia de cointegración, es decir, determinar si las variables presentan una relación en el largo plazo o que tiendan a un estado estacionario.

Ahora bien, cuando una serie de tiempo es no estacionaria y al diferenciarla se convierte en estacionaria, se dice que la serie es integrada de orden uno I (1). Por lo tanto, si se cuenta con ${ }^{n}$ series de tiempo que son I(1), se dice que presentan una relación de equilibrio en el largo plazo si existe una combinación lineal entre ellas que sea estacionaria o integrada de orden cero I $(0)$. 
Para demostrar fehacientemente que las $n$ series de tiempo cointegran, se utiliza el método de Johansen.

Se tiene la siguiente relación:

$$
y_{t}=\alpha_{1} \times y_{t-1}+\cdots+\alpha_{k} \times y_{t-k}+u_{t}
$$

Donde $y_{t}$ y sus rezagos son vectores $(n \times 1), u_{t}$ es un vector de $(n \times 1)$ que contiene errores ruido blanco, y $\alpha_{i}$ es una matriz $(n \times n)$. Asimismo, $n$ es el número de variables endógenas de la relación y $k$ es el número de rezagos.

En línea con Lahura y Vargas (2013), la ecuación (12) se puede re-escribir como un modelo vectorial de corrección de errores, llamado VECM:

$$
\Delta y_{t}=L_{1} \times \Delta y_{t-1}+\cdots+L_{k-1} \times \Delta y_{t-(k-1)}+T \times y_{t-k}+u_{t}
$$

Donde:

$$
L_{i}=-\left(\sum_{j=i+1}^{k} \alpha_{j}\right) \text { y } T=\left(\sum_{j=1}^{k} \alpha_{j}\right)-I_{r}
$$

En la ecuación (13), $y_{t}$ está en niveles y es I(1). Además, se analiza el rango de la matriz $T$ para determinar $(n-1)$ vectores de cointegración.

Johansen y Juselius (1990) desarrolla la prueba de la traza y la prueba de máxima verosimilitud para determinar el número de vectores de cointegración $r$. A continuación, se expresan las pruebas:

$$
\begin{aligned}
& \beta_{\text {traza }}=-R \times\left(\sum_{i=1}^{r} \ln \left(1-\beta_{i}\right)\right) \\
& \beta_{\text {máxima verosimilitud }}(r, r+1)=-R \times \ln \left(1-\beta_{r+1}\right)
\end{aligned}
$$

Dado los resultados, de encontrarse al menos un vector de cointegración, se podrá decir que las series se mueven juntas y presentan una relación de equilibrio a largo plazo. Así, se estimará un VECM para cuantificar la relación entre las variables (Court y Williams, 2011). Cabe indicar que los autores Nzengue y Faycal (2011) señalan que estimar un VAR en diferencias es incorrecto, ya que la mayoría de variables económicas y financieras no son estacionarias, por lo tanto, la forma ideal es estimar un 
VECM para establecer relaciones de corto y largo plazo; si observamos la ecuación 13, las matrices de la variable en diferencia expresan las relaciones de corto plazo y la matriz de la variable en niveles representan el largo plazo.

\section{Marco teórico}

\subsection{Antecedentes}

El teorema de Modigliani y Miller establece que existe un mundo sin imperfecciones en información y con mercados completos. Es decir, todas las empresas cuentan con instrumentos financieros para sus diferentes necesidades, por lo tanto, no interesa la estructura de financiamiento de la empresa.

Este es un buen inicio que sustenta la importancia del canal de préstamos bancarios en la economía, ya que no es cierto que las empresas cuenten con información completa y que tengan a su disposición diferentes productos para cubrir todas sus necesidades financieras. En economías emergentes como la peruana, los mercados de intermediación directa no están desarrollados (Armas, Castillo y Vega, 2014). Además, las micro y pequeñas empresas, así como, las medianas empresas dependen en su mayoría del financiamiento por parte de las entidades bancarias (Viladecut y Cabello, 2014). Bajo este enfoque de análisis, la política monetaria es relevante para poder, a través de los préstamos bancarios, influir en el producto y, por ende, en los precios.

El canal de préstamos bancarios es un mecanismo de transmisión de política monetaria. Es decir, a través del encaje bancario, se podría afectar el volumen de préstamos en la economía y, a su vez, impactar en el nivel de actividad económica agregada.

Particularmente, la relación del PBI per cápita en el Perú con la profundización financiera ${ }^{5}$, que se observa en la Figura 1, presenta una relación lineal en su comportamiento. Esta relación no es un sustento de que el crédito pueda exacerbar la actividad económica, ya que puede deberse a muchos factores; sin embargo, es una buena justificación para determinar la existencia del canal de préstamos bancarios en el Perú. 
En síntesis, el canal de préstamos bancarios funciona cuando la autoridad monetaria realiza una operación de mercado abierto, por ejemplo, compra de bonos a las entidades bancarias, lo cual se traduce en un aumento de las reservas o encajes en las cuentas de los bancos que tienen en el BCRP, ya que al pagarles se incrementa dicha cantidad. Con esto, las entidades bancarias cuentan con mayores fondos, lo cual permite incrementar los depósitos y, a su vez, poder ofertar mayor cantidad de préstamos bancarios. Así, las empresas al tener más fuentes de liquidez, podrían incentivar o dinamizar la actividad económica a través de un mayor nivel de inversión y consumo.

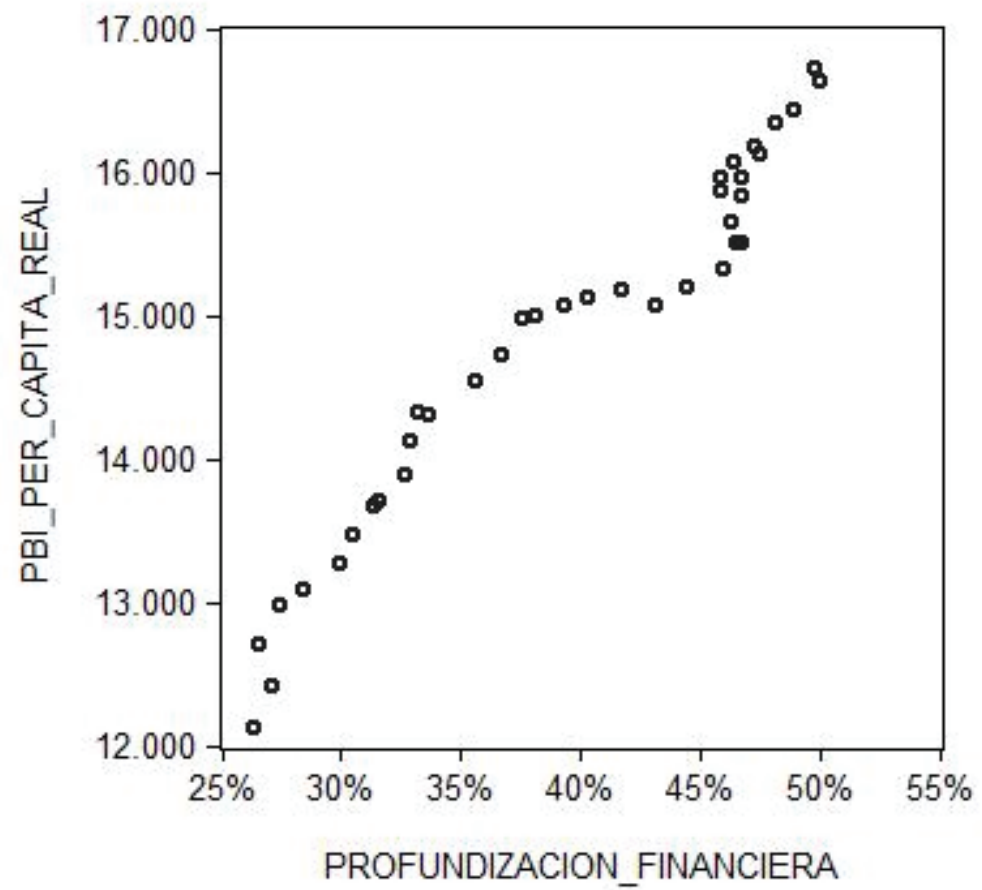

Figura 1. Relación entre el PBI per cápita y la profundización financiera

Fuente: Elaboración propia

Notas: Se presenta la relación entre el PBI per cápita real (PBI real 2007/población) en miles de nuevos soles y la profundización financiera 
(créditos del sector bancario/PBI real2007), datos desde el primer trimestre de 2010 hasta el primer trimestre de 2019.

\subsection{Modelo teórico}

Siguiendo la especificación del canal de préstamos bancarios explicada por De Gregorio (2007), y tomando como referencia a los creadores Bernanke y Blinder (1988), se plantea un modelo IS-LM con sector bancario.

En este modelo las entidades financieras tienen tres tipos de activos: dinero, bonos y préstamos. Por su parte, tienen un solo pasivo; los depósitos. Las entidades bancarias, tienen fondos de encaje en la autoridad monetaria y son representados por un porcentaje de los depósitos. Asimismo, los depósitos no pagan intereses al público. Todo el circulante o liquidez en la economía está representado por los depósitos. La diferencia entre los depósitos y los fondos de encaje representan el disponible de los bancos para ser invertidos en colocaciones o en comprar bonos. Así, los bancos destinarán más dinero a préstamos si la tasa de éstos sube en el mercado; de manera similar, si la tasa de mercado de los bonos sube, entonces, los bancos destinarán más dinero a comprar bonos. En este modelo, los bonos y los préstamos son sustitutos imperfectos ya que las tasas son diferentes. Cabe precisar que el mercado monetario está representado por la clásica LM. Finalmente, la IS estará representada en un inicio por el consumo, la inversión (depende de la tasa de los bonos y de la tasa activa) y el gasto público. Luego, será transformada por la curva CC (commodity and credit) que contiene a los préstamos bancarios.

A continuación, se detalla la relación entre las variables:

Las entidades bancarias tienen depósitos $(D)$, asimismo la autoridad monetaria exige una tasa de encaje bancario $(\theta)$, la cual se asumirá que es constante. Por lo tanto, los fondos de encaje $(f e)$ en las cuentas de la autoridad monetaria serán:

$$
f e=\theta \times D
$$

Asimismo, el disponible de los bancos es $(1-\theta) \times D$. Una parte será para colocaciones $\delta \times((1-\theta) \times D)$ y, la otra parte, será para invertir en bonos $(1-\delta) \times((1-\theta) \times D)$. Las cantidades a destinar en préstamos estarán en función de las tasas que cobran los bancos por los préstamos $(\tau)$ y por 
las tasas de rendimiento de los bonos del gobierno (i). Es decir, si las tasas de mercado por los préstamos aumentan, entonces, los bancos destinarán más a colocaciones; por otro lado, si la tasa de los bonos sube, los bancos querrán destinar menos dinero a colocaciones y más a invertir en bonos. De este modo, se presenta la siguiente relación:

$$
\delta=F\left(\tau^{+}, i^{-}\right)
$$

Entonces, la oferta de créditos de los bancos $\left(P^{S}\right)$ será representada por la siguiente ecuación:

$$
P^{s}=\delta\left(\tau^{+}, i^{-}\right) \times((1-\theta) \times D)
$$

Sin embargo, nos interesa observar el comportamiento de los fondos de encaje ( $f e$ ) sobre la oferta de préstamos. Así, de la ecuación (16), los depósitos son $\mathrm{D}=f e / \theta$, por lo tanto, reemplazamos en la ecuación (18) y obtenemos la siguiente ecuación:

$$
P^{s}=\delta\left(\tau^{+}, i^{-}\right) \times \frac{(1-\theta)}{\theta} \times f e
$$

La demanda de préstamos estará en función de la actividad económica ( y), de la tasa que cobran los bancos $(\tau)$ y de la tasa de los bonos (i). No obstante, sólo vamos a considerar a la tasa activa $(\tau)$ ya que los mercados de intermediación directa en Perú no están desarrollados (Armas, Castillo y Vega, 2014).

$$
P^{d}=\mathrm{F}\left(\tau^{-}, y^{+}\right)
$$

Cabe precisar, que si la tasa de los préstamos $(\tau)$ sube, entonces, la demanda de préstamos $\left(P^{d}\right)$ caerá; por otro lado, si la actividad económica (y) se incrementa, la demanda por créditos $\left(P^{d}\right)$ subirá.

En términos ideales, el equilibrio en el mercado de créditos estará representado por:

$$
F\left(\tau^{-}, y^{+}\right)=\delta\left(\tau^{+}, i^{-}\right) \times \frac{(1-\theta)}{\theta} \times f e
$$

Por otro lado, la IS estará representado por (y), que será la demanda agregada. También, es la suma del consumo $(C)$, la inversión $(I)$ y el gasto 
del gobierno $(G)$. A su vez, la $(I)$ depende de $\tau$ e $i$. Con ello, la demanda agregada estará en función de $\tau$ e $i$ en forma negativa, ya que cualquier incremento de las tasas desincentivaría la actividad económica.

$$
y=F\left(\tau^{-}, i^{-}\right)
$$

La LM estará representada por la clásica ecuación del dinero. Se asume que todo el circulante son los depósitos:

$$
D^{d}=D\left(i^{-}, y^{+}\right)
$$

De la ecuación (21), si la tasa de interés de los bonos (i) sube, entonces los bancos destinarán más dinero para comprar bonos, por lo tanto, la oferta de los préstamos caerá, lo cual podría incrementar la tasa de los préstamos $(\tau)$. De otro lado, un aumento de la actividad económica $(y)$ incrementa la demanda de préstamos, así la tasa de los préstamos $(\tau)$ podría subir. Finalmente, un incremento de los fondos de encaje de los bancos $(f e)$ incrementa la oferta de préstamos y, por ende, la tasa de los préstamos $(\tau)$ cae. Bajo este enfoque de análisis, podemos obtener ecuación (24):

$$
\tau=F\left(i^{+}, y^{+}, f e^{-}\right)
$$

Finalmente, reemplazamos la ecuación (24) en la ecuación (22) y obtenemos lo siguiente:

$$
y=F\left(i^{-}, f e^{+}\right)
$$

Si la tasa de los bonos (i) se incrementa, entonces, los bancos destinarán menos dinero para ofertar como préstamos (ya que preferirán comprar más bonos), esto incrementará la tasa de créditos $(\tau)$ en el mercado, con lo cual desincentivaría la demanda agregada. Por otro lado, si los bancos tienen más fondos de encaje agregado, esto permitirá incrementar la base de los depósitos, con lo cual tendría un impacto positivo en la oferta de préstamos y, por ende, ocasionaría una disminución en la tasa activa $(\tau)$. Así, estos movimientos estimularían a la demanda agregada (y). De este modo, la IS se convierte en la CC representada por la ecuación (25).

En este contexto, es de suma importancia que los bancos no cuenten con fondos adicionales a los depósitos bancarios $(D)$. 


\subsection{Revisión de la literatura Internacional}

Kassem y Awdeh (2018) estudian cómo la política monetaria puede afectar la oferta de préstamos de los bancos para el Líbano desde el periodo que va desde enero de 1992 hasta diciembre de 2016. Usando como variable dependiente al nivel de créditos agregados $\mathrm{y}$, como variables independientes, al capital de los bancos, oferta monetaria (M2), la tasa de interés pasiva y los depósitos de los no residentes (sabiendo que todas las variables utilizadas son no estacionarias), encuentran que a través del uso de la metodología VECM sólo la oferta monetaria afecta en el largo plazo a la cantidad de préstamos ofertados.

Yarasevika, Tongato y Muthia (2015) analizan el canal de préstamos bancarios para la economía de Indonesia a través de un enfoque VECM. En particular, el periodo de análisis es de frecuencia anual que va desde 1986 hasta el 2013. Para tal objetivo, sólo toman una relación teórica, donde establecen que los préstamos bancarios (BL) están en función con la tasa activa (RC), el índice de precios al consumidor (CPI), el PBI y un ratio de requerimiento de reservas (RR). A través del método aumentado de Dickey-Fuller (ADF) encuentran que todas las variables son I(1). Asimismo, aplican criterios de información para determinar el número de rezagos óptimos. Finalmente, utilizando la prueba de la traza y de máxima verisimilitud de Johansen y Juselius (1990) encuentra un vector de cointegración, lo cual confirma la existencia del canal de préstamos bancarios para la economía de Indonesia. De este modo, se aplica un VECM. Así, en el largo plazo, un continuo crecimiento del PBI en 1\% causa un incremento de los préstamos en $6.82 \%$ y un incremento del $1 \%$ en RR produce un incremento de los créditos en $2.7 \%$.

Sun, Gan y Hu (2010) confirman la existencia del canal de préstamos bancarios en China durante el periodo que va desde el primer trimestre de 1997 hasta el cuarto trimestre de 2008. Para evitar el problema de identificación de la oferta y demanda de préstamos bancarios, estiman un VECM con restricciones siguiendo a Mello y Pisu (2009). De esta manera, establecen que la oferta de préstamos bancarios $\left(L^{s}\right)$ está en función de un ratio de requerimiento de reserva (RR), de la tasa de préstamos bancarios (RC) y de la tasa de inflación $(\boldsymbol{\pi})$. Por otro lado, la demanda de préstamos $\left(L^{d}\right)$ está en función del PBI, RC y $\pi$. Cabe precisar que estas relaciones 
son parecidas a las ecuaciones (19) y (20) propuestas en el modelo teórico desarrollado por Bernanke y Blinder (1988). En la ecuación de largo plazo, encuentran que el PBI es un determinante fuerte de la demanda de préstamos; un incremento de $1 \%$ en el PBI causa un incremento de $0.8 \%$. Por su parte, un incremento de $1 \%$ en el ratio de requerimiento de reserva, afecta negativamente a la oferta de préstamos en $0.098 \%$.

Mello y Pisu (2009) determinan la existencia del canal de préstamos bancarios en Brasil usando datos mensuales agregados que van desde diciembre de 1995 hasta junio de 2008. La metodología empleada es el VECM. A través de restricciones, identifican una ecuación para la oferta y demanda de préstamos. De esta manera, la oferta de créditos está en función del patrimonio de los bancos $(C)$, de la inflación $(\pi)$, de la tasa interbancaria $\left(r_{b}\right)$ y de la tasa activa $\left(r_{l}\right)$. Por su parte, la demanda de los préstamos está en función de $(\pi)$, el PBI y $\left(r_{l}\right)$. Dado los resultados en el largo plazo, un incremento permanente de $1 \%$ de $\left(r_{l}\right)$ causa un incremento de $0.040 \%$ de la oferta de préstamos y un incremento de $1 \%$ en $\left(r_{b}\right)$ causa una disminución de la oferta de préstamos en $0.097 \%$. Finalmente, en el corto plazo, la oferta de préstamos es corregida por $\left(r_{b}\right)$, determinando de esta manera la importancia de la política monetaria sobre el canal de préstamos en Brasil.

\subsection{Revisión de la literatura Nacional}

Bustamante, Cuba y Nivin (2019) utilizando datos nivel de bancos de Perú, estudian cómo la política monetaria, a través de la tasa de interés y el requerimiento de reservas, afecta la oferta de préstamos. Para créditos en moneda nacional, los bancos más capitalizados, con elevados ratios de liquidez, con bajo nivel de riesgos y con más utilidades tienden a otorgar más préstamos. También, cuando el BCRP establece una política monetaria restrictiva, ésta sería débil en los bancos que poseen más activos y tienen altos niveles de liquidez. Finalmente, el requerimiento de reservas en moneda nacional y extranjera es efectivo en frenar la oferta de préstamos bancarios, lo cual otorga un soporte a las políticas de encaje bancario para suavizar el ciclo crediticio.

Vega y Chávez (2017) analizan la propagación de choques de requerimiento de encaje en moneda nacional y extranjera para el Perú utilizando la metodología GVAR (modelo de vectores autorregrasivos global) 
con data individual y agregada. Los resultados muestran evidencia que una política contractiva de encaje causa una reducción, en el muy corto plazo, en el crédito en moneda nacional y extranjera, el PBI y los precios, sin embargo, los fondos de encaje aumentan. Finalmente, consideran que la política de requerimientos de encaje es efectiva sobre el nivel de créditos agregados en moneda nacional y, podría serlo, para los créditos agregados en moneda extranjera.

Pérez y Vega (2014) identifican cómo los choques de la política monetaria convencional y de tasas de encaje en soles y dólares afectan el nivel de créditos en la economía peruana durante el periodo 1995-2013. En particular, a través de la metodología SVAR (modelo de vectores autorregresivos estructurales) con restricciones, encuentran que un incremento de la tasa de requerimiento de encaje en soles afecta negativamente a los créditos en soles y dólares. Asimismo, un incremento de la tasa de encaje en moneda extranjera sólo reduce los préstamos en dólares. Finalmente, resaltan que la política monetaria no convencional, encajes en soles y dólares, son un buen complemento de la política monetaria de tasas de interés.

Viladecut y Cabello (2014) estudian el canal de créditos en el Perú durante el periodo que va desde el primer trimestre de 2002 hasta el segundo trimestre de 2012. La metodología empleada es un SVAR con restricciones y expectativas. La ecuación utilizada coloca como variable dependiente a la tasa de crecimiento de crédito, la cual está en función de los rezagos del PBI, tasa de interés, tipo de cambio y crédito. Encuentra que la tasa de crecimiento del crédito se ve impactada negativamente por la tasa de interés nominal y positivamente por los rezagos del PBI. Finalmente, concluyen que el canal de préstamos bancarios estaría operando.

Carrera (2011) estudia el canal de préstamos bancarios a través de la metodología SVAR utilizando data a nivel de bancos durante el periodo 2002-2010. En sus resultados, encuentra que la política monetaria, tasa de interés corto de plazo, mantiene una relación inversa con el crecimiento del crédito; y, una relación directa, entre el crecimiento de los créditos con las características de los bancos a través de la política monetaria convencional. No obstante, el canal no sería cuantitativamente relevante como mecanismo de transmisión para las variables de la actividad económica en el Perú. 
En síntesis, no existe un estudio formal del canal de préstamos bancarios en el Perú donde se pueda ver la existencia en el largo plazo. Además, como lo sugiere Nzengue y Faycal (2011) si las variables son I(1) se debe usar la metodología VECM, la cual permite ver las relaciones, interacciones y choques en el corto y largo plazo. En línea con lo discutido en Carrera (2011), sugiere robustecer los resultados con técnicas de cointegración, es decir, estudiar el largo plazo. Cabe precisar que los estudios a nivel de características de los bancos han cobrado relevancia a partir del trabajo de los autores Ehrmann y otros (2001), empero dichos estudios parten de la ecuación (19) y (20) propuesta por Bernanke y Blinder (1988).

\section{Resultados y discusión}

\subsection{Datos y hechos estilizados}

Las series a utilizar son de frecuencia trimestral que abarcan el periodo que va desde el primer trimestre de 2010 hasta el primer trimestre de 2019. Son obtenidas de la página web del BCRP. En este trabajo se usa los créditos del sistema bancario al sector privado en moneda nacional $(\mathrm{Cmn})$, en moneda extranjera ( $\mathrm{Cmex}$ ), la tasa activa en moneda nacional (Tamn), en moneda extranjera (Tamex), el rendimiento del bono del gobierno peruano a 10 años en soles ( $i$ ), el rendimiento del bono de USA a 10 años ( $i^{*}$ ), los fondos de encaje en moneda nacional ( $f e m n$ ), en moneda extranjera (femex) y el PBI real. Cabe precisar que para el cálculo de (femn) se utilizó un promedio de la tasa de encaje en moneda nacional (10\%) multiplicado por el total de obligaciones sujetas a encaje en moneda nacional (TOSEMN); de la misma manera, para calcular (femex) se usó un promedio de la tasa de encaje en moneda extranjera (38\%) multiplicado por el total de obligaciones sujetas a encaje en moneda extranjera (TOSEME). En términos de secuencia lógica, el promedio utilizado es para evitar la volatilidad en las series, tal como lo hizo Viladecut y Cabello (2014) para el cálculo del crédito total a través de un tipo de cambio fijo.

La Figura 2 muestra la evolución de las series agregadas a utilizar en el modelo teórico. Nótese que $\mathrm{Cmn}$, femn y el PBI muestran una tendencia alcista. Por su parte, Cmex presenta una tendencia alcista que va desde el primer trimestre de 2010 hasta el segundo trimestre de 2014, mientras que 
desde el tercer trimestre de 2014 hasta el primer trimestre de 2016 muestra una clara tendencia bajista, no obstante desde el segundo trimestre de 2016 hasta el primer trimestre de 2019 ha tenido una recuperación leve, sin alcanzar máximos anteriores. Asimismo, femex presentan una tendencia alcista que va desde el primer trimestre de 2010 hasta el tercer trimestre de 2015, mientras que desde el cuarto trimestre de 2015 hasta el primer trimestre de 2019 presenta una tendencia bajista leve. Este comportamiento en moneda extranjera podría ser por el Programa de Desdolarización financiera de 2013 que ha tenido impacto sobre el traspaso de créditos en dólares por soles en los bancos (Contreras y otros, 2019). Finalmente, la Tamn presenta una tendencia bajista parecida al comportamiento de la Tamex.

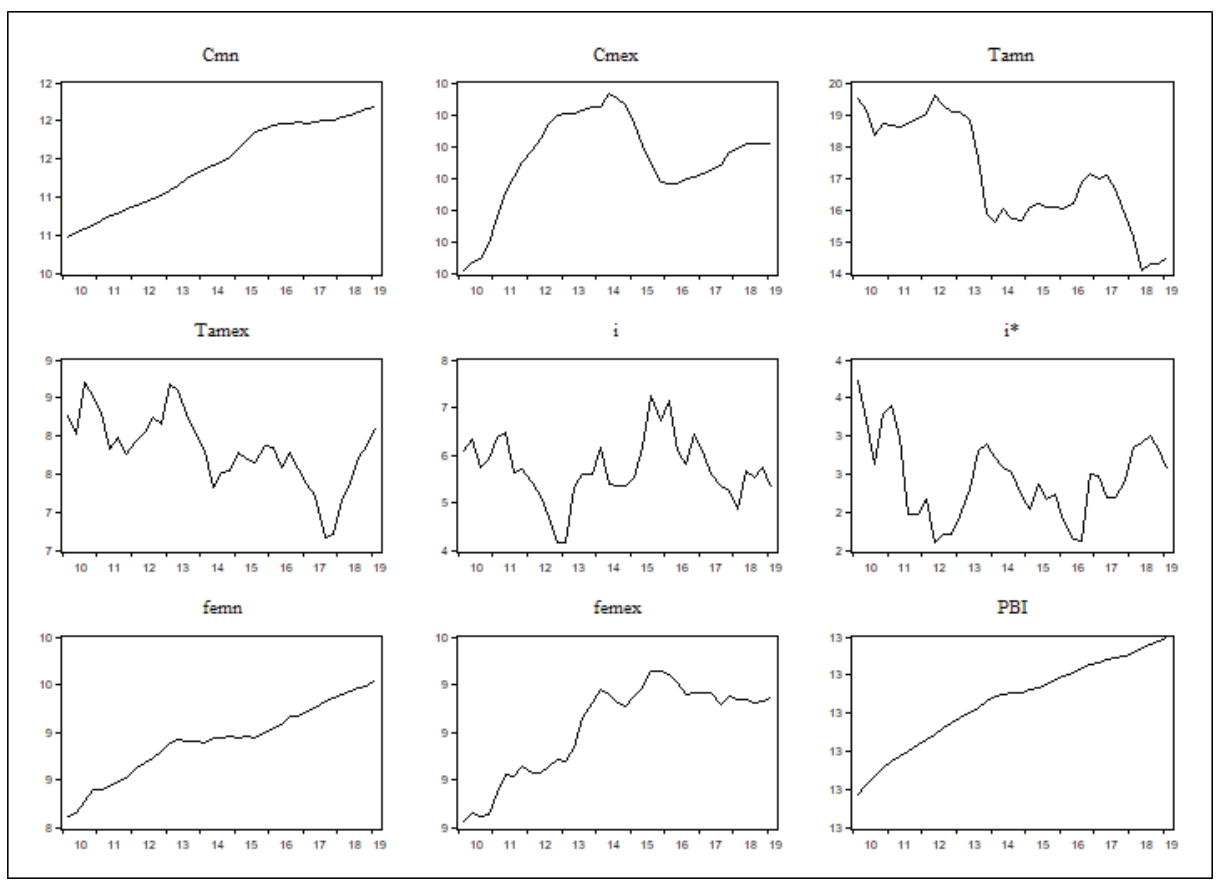

Figura 2. Comportamiento de series agregadas

Fuente: Elaboración propia.

Notas: El eje vertical representa cantidad y el eje horizontal el tiempo. Cmn, Cmex, femn, femex y PBI se encuentran en logaritmos y se aplicó 
la metodología Census X-12. Por otro lado, Tamn, Tamex, i y i* se encuentran en porcentajes.

\subsection{Estacionariedad de las series}

Para realizar un análisis de cointegración, las series deben ser I(1). Para esto aplicaremos la prueba ADF desarrollada en la metodología de la investigación.

$$
\begin{aligned}
& H_{0}: \delta^{*}=0 \\
& H_{1}: \delta^{*}<0
\end{aligned}
$$

En tal sentido, debemos de rechazar $H_{0}$ de raíz unitaria para para confirmar la estacionariedad de las series. De este modo, aplicamos el p-values de la prueba. Así, si éste resulta menor a 5\% se rechazaría la $H_{0}$. Donde, $N$ es la serie en niveles y, $d$ es la serie en primera diferencia.

Tabla 1

Prueba ADF de las series

\begin{tabular}{|c|c|c|c|c|c|c|c|c|c|}
\hline & Cmn & Cmex & Tamn & Tamex & $i$ & $i^{*}$ & femn & femex & PBI \\
\hline$N$ & 0,89 & 0,79 & 0,28 & 0,61 & 0,48 & 0,24 & 0,39 & 0,86 & 0,30 \\
\hline$d$ & 0,09 & 0,01 & 0,00 & 0,00 & 0,00 & 0,00 & 0,02 & 0,00 & 0,04 \\
\hline
\end{tabular}

Fuente: Elaboración propia.

Notas: Para calcular los p-values se utilizó el programa econométrico Eviews 8.

El Tabla 1 nos muestra los p-values de la prueba ADF de las series en niveles y en diferencias. Cabe precisar que la hipótesis nula de la primera diferencia de $\mathrm{Cmn}$ se rechaza a un nivel de 10\%. En términos generales, todas las series son I (1).

\subsection{Especificación del modelo teórico}

Primero, tenemos que representar la ecuación (19) para ver cómo los fondos de encaje afectan a la oferta de préstamos. Segundo, establecer la ecuación (25), que representa la curva CC, para ver cómo los fondos de encaje afectan a la actividad económica. En tal sentido, el Perú 
es una economía parcialmente dolarizada, por lo tanto se tiene que realizar un análisis por separado en moneda nacional y extranjera (Bustamante, Cuba y Nivin, 2019; Vega y Chávez, 2017; Pérez y Vega, 2014). Finalmente, antes de realizar estos pasos, se debe de hacer un análisis de causalidad a lo granger para ver si realmente las variables independientes son buenos predictores de la variables dependientes (Kassem y Awdeh, 2018; Carrera, 2011; Bringas y Tuesta, 1997).

\subsubsection{Especificación del modelo teórico en moneda nacional}

Vamos a partir de la ecuación (19) y (25), la cual estarán representadas por las siguientes funciones en moneda nacional:

$$
\begin{aligned}
& \text { Cmn }=F(\text { Tamn }, i, \text { femn }) \\
& P B I=F(i, \text { femn })
\end{aligned}
$$

El Tabla 2 nos muestra el test de granger para la ecuación (26). Los femn son buenos predictores en el largo plazo de los $C m n$, y viceversa. Asimismo, los femn son buenos predictores de la Tamn. Carrera (2011) encontró que la dirección de predictibilidad va desde la política monetaria (tasa de interés interbancaria) hacia la calidad del crédito. Así, los resultados van en línea con lo dicho anteriormente, con la diferencia que nuestro instrumento de política monetaria sería la cantidad de fondos de encaje en moneda nacional. Además, podríamos afirmar que la relación es viceversa. Esto se podría interpretar que la autoridad monetaria está utilizando activamente el instrumento de política monetaria no convencional, tasa de requerimiento de encaje en soles, para suavizar el ciclo crediticio (Vega y Chávez, 2017; Pérez y Vega, 2014).

Tabla 2

Test de Granger para Cmn, Tamn, i y femn

\begin{tabular}{|l|l|}
\hline Hipótesis nula & p-values \\
\hline$i$ no causa a lo granger a Cmn & 0,0022 \\
\hline Cmn no causa a lo granger a $i$ & 0,0630 \\
\hline femn no causa a lo granger a Cmn & 0,0210 \\
\hline Cmn no causa a lo granger a femn & 0,0316 \\
\hline femn no causa a lo granger a Tamn & 0,0112 \\
\hline Cmn no causa a lo granger a Tamn & 0,0405 \\
\hline
\end{tabular}

Fuente: Elaboración propia. 
Notas: Si $p$-values es menor a $5 \%$ se rechaza la hipótesis nula. Para calcular los $p$-values se utilizó el programa econométrico Eviews 8. Asimismo, para calcular los rezagos óptimos se utilizó un VAR, donde a través de los criterios de información se determinó 2 rezagos, tal como lo sugiere Lahura (2011).

El autor Carrera (2011) sugiere reforzar el ejercicio de la identificación de la existencia del canal de préstamos bancarios en el Perú, como mecanismo de transmisión de la política monetaria, usando técnicas de cointegración. En ese sentido, para ver si existe una relación de equilibrio en el largo plazo, se debe utilizar el método de Josansen (Kassem y Awdeh, 2018; Yarasevika, Tongato y Citra, 2015; Sun, Gan y Hu, 2010; Mello y Pisu, 2009).

Tabla 3

Test de Johansen en moneda nacional para Cmn, Tamn, i y femn

\begin{tabular}{|l|l|l|l|l|l|}
\hline \multicolumn{1}{|c|}{ Data tren } & \multicolumn{1}{c|}{ None } & \multicolumn{1}{c|}{ None } & \multicolumn{1}{c|}{ Linear } & \multicolumn{1}{c|}{ Linear } & \multicolumn{1}{c|}{ Quadratic } \\
\hline Test Type & $\begin{array}{l}\text { No intercept / No } \\
\text { trend }\end{array}$ & $\begin{array}{l}\text { Intercept / No } \\
\text { trend }\end{array}$ & $\begin{array}{l}\text { Intercept / No } \\
\text { trend }\end{array}$ & Intercept/Trend & Intercept/Trend \\
\hline Prueba Traza & 0 & 2 & 1 & 1 & 1 \\
\hline $\begin{array}{l}\text { Prueba Máxima } \\
\text { verosimilitud }\end{array}$ & 0 & 0 & 0 & 0 & 1 \\
\hline
\end{tabular}

Fuente: Elaboración propia.

Notas: Para calcular los rezagos óptimos se utilizó un VAR, donde a través de los criterios de información se determinó 2 rezagos, tal como lo sugiere Lahura (2011). Los números representan a la cantidad de vectores de cointegración. Finalmente, se utilizó el programa econométrico Eviews 8 para realizar la prueba.

El ejercicio de la tabla 3, da luz para concluir que probablemente exista una relación de largo plazo entre las series propuestas, a través de la prueba de la traza, se está afirmando fehacientemente la cointegración en moneda nacional. Una posible interpretación, de la mano de la teoría propuesta por Bernanke y Blinder (1988), sería que los bancos al acumular más dinero como fondos de reservas en la autoridad monetaria permiten en el largo plazo poder ampliar las colocaciones. No obstante, en el corto plazo podría tener efecto la política monetaria no convencional, tasas de encaje en soles, 
como un mecanismo de corrección. Los resultados son sugerentes para una primera identificación del canal de préstamos bancarios en moneda nacional. Así Kassem y Awdeh (2018) encuentran a través del método de Johansen un vector de cointegración entre las series crédito, capital, depósitos, M2 e interés en el Líbano. Por su parte, Sun, Gan y Hu (2010) encontraron un vector de cointegración para el canal de préstamos en la economía China. De la misma manera, Mello y Pisu (2009) encontraron una relación de largo plazo para la economía de Brasil.

En línea con los autores Court y Williams (2011), una vez que se ha determinado la existencia de cointegración ya se podría utilizar el modelo de corrección de errores VECM ${ }^{6}$.

Por lo tanto, la ecuación (26) estará representada numéricamente de la siguiente forma en el largo plazo:

$$
\text { Cmn }=12,85_{(0,0000)}+0,09_{(0,0012)} \text { Tamn }+0,39_{(0,0000)} i+2,22_{(0,0000)} \text { femn }
$$

Los subíndices representan los $p$-values de los coeficientes de las variables. Como observamos resultan altamente significativos. En tal sentido, refuerza nuestra investigación. En línea con la explicación de Lahura y Vega (2011) para un VECM, podemos decir que, en equilibrio, si los fondos de encaje en moneda nacional femn se incrementan en un punto porcentual los créditos en moneda nacional $\mathrm{Cmn}$ aumentan en 2.22 por ciento. Asimismo, si la tasa de los préstamos bancarios Tamn se incrementan en un punto porcentual (por ejemplo de 19\% a 20\%) los créditos en moneda nacional Cmn aumentan en 0.09\%. Estos resultados están en línea con la teoría propuesta por Bernanke y Blinder (1988) y explicada por el autor De Gregorio (2007). Es decir, cuando la autoridad monetaria realiza una operación de mercado abierto, por ejemplo, comprar bonos, los bancos incrementan sus fondos de reserva, por lo tanto, incrementan sus bases para realizar más préstamos. Por su parte, si la tasa de los préstamos aumenta en el mercado esto incentiva a los bancos a querer ofertar más préstamos. Por otro lado, Sun, Gan y Hu (2010) encuentran que un incremento en $1 \%$ en el ratio de reservas disminuye la oferta de préstamos en $0.098 \%$ para la economía China. Sin embargo, si se incrementa la tasa de encaje, se considera una política monetaria contractiva y, lo que se espera, es reducir los préstamos. Al igual que, Mello y Pisu (2009), encuentran 
que una política monetaria contractiva, aumento de la tasa interbancaria en $1 \%$, produce una disminución en la oferta de préstamos en Brasil de 0.097\%; por su parte, un incremento de la tasa de los préstamos bancarios en $1 \%$, aumenta los créditos en $0.04 \%$.

A diferencia de Su, Gan y Hu (2010) y Mello y Pisu (2009), estos autores no llegan a conectar el instrumento de política monetaria sobre el producto. Esta es la razón del porqué no colocamos restricciones en el modelo de corrección de errores VECM. Por lo tanto, esta investigación sí logra conectar los fondos de encaje con el PBI, tal como lo sugiere la teoría.

La Tabla 4 nos muestra el test de causalidad de granger para la ecuación (27). Particularmente, los fondos de encaje en soles femn son buenos predictores de la actividad económica a un nivel de significancia del $10 \%$. Cabe señalar que esta prueba sería un buen ejercicio antes de encontrar alguna relación en política monetaria (Bringas y Tuesta, 1998).

Tabla 4

Test de Granger para PBI. i, femn

\begin{tabular}{|l|l|}
\hline Hipótesis nula & p-values \\
\hline femn no causa a lo granger a PBI & 0,0525 \\
\hline
\end{tabular}

Fuente: Elaboración propia.

Notas: Si $p$-values es menor a $10 \%$ se rechaza la hipótesis nula. Para calcular los $p$-values se utilizó el programa econométrico Eviews 8. Asimismo, para calcular los rezagos óptimos se utilizó un VAR, donde a través de los criterios de información se determinó 1 rezago, tal como lo sugiere Lahura (2011).

Vamos a aplicar el test de Johansen para la ecuación (27). La Tabla 5 nos muestra que probablemente exista un vector de cointegración, con lo cual se está demostrando que existe una relación de equilibrio en el largo plazo. La ecuación (27) representa la curva IS con sector bancario, denominada por Bernanke y Blinder (1988) como la curva CC. Una posible interpretación de este resultado, de acuerdo con la teoría, es que los mecanismos de transmisión de la política monetaria, a través del canal de los préstamos en moneda nacional, estaría operando en la economía peruana. 
Tabla 5

Test de Johansen para PBI. i, femn

\begin{tabular}{|l|l|l|l|l|l|}
\hline \multicolumn{1}{|c|}{ Data trend } & \multicolumn{1}{c|}{ None } & \multicolumn{1}{c|}{ None } & \multicolumn{1}{c|}{ Linear } & \multicolumn{1}{c|}{ Linear } & \multicolumn{1}{c|}{ Quadratic } \\
\hline Test Type & $\begin{array}{l}\text { No intercept / } \\
\text { No trend }\end{array}$ & $\begin{array}{l}\text { Intercept / No } \\
\text { trend }\end{array}$ & $\begin{array}{l}\text { Intercept / No } \\
\text { trend }\end{array}$ & $\begin{array}{l}\text { Intercept / } \\
\text { Trend }\end{array}$ & $\begin{array}{l}\text { Intercept / } \\
\text { Trend }\end{array}$ \\
\hline Prueba Traza & 0 & 0 & 1 & 1 & 1 \\
\hline $\begin{array}{l}\text { Prueba Máxima } \\
\text { verosimilitud }\end{array}$ & 0 & 1 & 1 & 1 & 1 \\
\hline
\end{tabular}

Fuente: Elaboración propia.

Notas: Para calcular los rezagos óptimos se utilizó un VAR, donde a través de los criterios de información se determinó 1 rezago. Los números representan la cantidad de vectores de cointegración. Finalmente, se utilizó el programa econométrico Eviews 8 para realizar la prueba.

En línea con Court y Williams (2011), ya podríamos evaluar el modelo de corrección de errores VECM para cuantificar la relación de largo plazo.

Por lo tanto, la ecuación (27), estará representada numéricamente de la siguiente forma en el largo plazo para la economía peruana:

$P B I=8,30_{(0,0000)}+0,03_{(0,0000)} i+0,50_{(0,0000)}$ femn $+0,004_{(0,0026)}$ Trend

Los subíndices representan el $p$-values de las variables. Como observamos resultan ser altamente significativos. En línea con la explicación de Lahura y Vega (2011), en equilibrio, si los fondos de encaje en moneda nacional femn se incrementan en un punto porcentual el PBI se incrementa en 0.50 puntos porcentuales. También, siguiendo la definición de Lahura y Vargas (2013), podemos decir que, un aumento permanente de un punto porcentual de los fondos de encaje en soles femn está asociado a un aumento permanente de $0.5 \%$ del PBI. En síntesis, si el Banco Central de Reserva del Perú BCRP realiza una operación de mercado abierto, donde las entidades bancarias puedan incrementar sus colocaciones en el mercado, esto producirá un incremento de la actividad económica en el Perú. Estos resultados van de la mano con la teoría propuesta. será:

En síntesis, el canal de los préstamos bancarios en moneda nacional

$\uparrow 1 \%$ femn $\rightarrow \uparrow 2,22 \% \mathrm{Cmn} \rightarrow \uparrow 0,5 \%$ PBI 


\subsubsection{Especificación del modelo teórico en moneda extranjera}

Vamos a partir de la ecuación (19) y (25), la cual estarán representadas por las siguientes funciones en moneda extranjera:

$$
\begin{aligned}
& \text { Cmex }=F\left(\text { Tamex }, i^{*}, \text { femex }\right) \\
& P B I=F\left(i^{*}, \text { femex }\right)
\end{aligned}
$$

La Tabla 6 indica que los femex son buenos predictores de los $C m e x$ . Este resultado se ve reforzado con lo encontrado por los autores Bustamante, Cuba y Nivin (2019), donde señalan que los requerimientos de reservas en moneda extranjera son efectivos en frenar los créditos.

Tabla 6

Test de Granger para Cmex, Tamex, $i^{*}$ y femex

\begin{tabular}{|l|l|}
\hline Hipótesis nula & p-values \\
\hline femex no causa a lo granger a Cmex & 0,0025 \\
\hline Cmex no causa a lo granger a femex & 0,0878 \\
\hline Tamex no causa a lo granger a femex & 0,0175 \\
\hline
\end{tabular}

Fuente: Elaboración propia.

Notas: Si $p$-values es menor a $5 \%$ se rechaza la hipótesis nula. Para calcular los $p$-values se utilizó el programa econométrico Eviews 8. Asimismo, para calcular los rezagos óptimos se utilizó un VAR, donde a través de los criterios de información se determinó 1 rezago, tal como lo sugiere Lahura (2011).

El test de Granger, sugerido por Vega y Chávez (2017) y Bringas y Tuesta (1998), nos permite evaluar una posible relación a largo plazo entre las variables. En tal sentido, al ser las series agregadas e I(1) aplicamos una prueba formal, como el test de Johansen, para observar si existe cointegración (Court y Williams, 2011).

La Tabla 7 nos muestra la prueba de Johansen. Se encuentra un vector de cointegración. Por lo tanto, la prueba de la traza afirma que existe una relación a largo plazo entre los femex y los Cmex. La relación encontrada va de la mano con la teoría propuesta por Bernake y Blinder (1988) 
y explicada por De Gregorio (1998). Estos resultados se ven reforzados por las medidas adoptadas por el BCRP para desdolarizar la economía peruana, con lo cual algunos bancos cambiaron préstamos en dólares por soles, sobretodo en el sector corporativo y pequeñas empresas (Contreras y otros, 2019).

Tabla 7

Test de Johansen en moneda extranjera para Cmex, Tamex, $i^{*}$ y femex

\begin{tabular}{|l|l|l|l|l|l|}
\hline Data trend & None & None & Linear & Linear & Quadratic \\
\hline Test Type & $\begin{array}{l}\text { No intercept/ } \\
\text { No trend }\end{array}$ & $\begin{array}{l}\text { Intercept / No } \\
\text { trend }\end{array}$ & $\begin{array}{l}\text { Intercept/ } \\
\text { No trend }\end{array}$ & Intercept/Trend & Intercept/ Trend \\
\hline Prueba Traza & 1 & 0 & 1 & 0 & 0 \\
\hline $\begin{array}{l}\text { Prueba Máxima } \\
\text { verosimilitud }\end{array}$ & 0 & 0 & 0 & 0 & 0 \\
\hline
\end{tabular}

Fuente: Elaboración propia.

Notas: Para calcular los rezagos óptimos se utilizó un VAR, donde a través de los criterios de información se determinó 1 rezago, tal como lo sugiere Lahura (2011). Los números representan la cantidad de vectores de cointegración. Finalmente, se utilizó el programa econométrico Eviews 8 para realizar la prueba.

Al igual que (Yarasevika, Tongato y Citra, 2015; Sun, Gun y Hu, 2010; Mello y Pisu, 2009), después de encontrar un vector de cointegración se procede a estimar un VECM para cuantificar la posible relación de largo plazo. En tal sentido, la ecuación (28) estará representada numéricamente de la siguiente forma:

Cmex $=33,78-0,01_{(0,9787)}$ Tamex $-0,50_{(0,0847)} i^{*}+4,89_{(0,0006)}$ femex

Observamos que los fondos en moneda extranjera femex son altamente significativos. Siguiendo la explicación de Lahura y Vargas (2013), podemos decir que un aumento permanente de un punto porcentual en femex esta asociado a un aumento permanente de 4,89\% de los Cmex. El alto porcentaje tiene su explicación porque el BCRP viene estableciendo desde el 2013 un programa de desdolarización en la economía peruana (Contreras y otros, 2019). Así, el primer trimestre de 2010 los créditos en dólares representaban el 49\% del total de créditos y, en el primer trimestre de 2019, representan 30\%. En síntesis, las medidas adoptadas por la entidad monetaria son 
eficientes para frenar el crédito en moneda extranjera, por lo tanto, este primer mecanismo de transmisión está funcionando.

La Tabla 8 nos muestra el Test de Granger para la ecuación (29), donde observamos que los femex no son buenos predictores en el largo de la actividad económica. Por lo tanto, no tendría validez, estadística y económica, para establecer una posible relación de largo plazo entre las variables ${ }^{7}$. Una posible interpretación de este resultado sería que las entidades bancarias no dependen de los depósitos para hacer préstamos en moneda extranjera, es decir, tienen fuentes alternativas.

Tabla 8

Test de Granger para PBI. $i^{*}$ y femex

\begin{tabular}{|l|l|}
\hline Hipótesis nula & p-values \\
\hline femex no causa a lo granger a $P B I$ & 0,7676 \\
\hline
\end{tabular}

Fuente: Elaboración propia.

Notas: Si $p$-values es menor a $5 \%$ se rechaza la hipótesis nula. Para calcular los $p$-values se utilizó el programa econométrico Eviews 8. Asimismo, para calcular los rezagos óptimos se utilizó un VAR, donde a través de los criterios de información se determinó 3 rezagos, tal como lo sugiere Lahura (2011).

Bernanke y Blinder (1988) definieron al canal de préstamos como un mecanismo donde la autoridad monetaria, a través de su política monetaria, puede afectar al volumen de préstamos y éstos pueden afectar la actividad económica. Bajo esta definición, el BCRP puede afectar, a través de su política de monetaria, el volumen de préstamos en moneda extranjera, empero éstos no afectan a la actividad económica en el Perú. Con ello, no existe en el Perú un canal de préstamos bancarios en moneda extranjera durante el periodo de análisis.

\section{Reflexiones finales}

Es sumamente importante tener claro la estrecha relación entre el sector bancario y la actividad económica. Este match ha cobrado relevancia después de la CFI, donde nuestro principal instrumento de política monetaria (tasa de interés interbancaria) se vio afectado, lo cual dificulta los 
mecanismos de transmisión de política monetaria. Más aún, que el Perú viene atravesando un proceso de expansión del crédito y, además, es una economía pequeña y abierta expuesta a los diferentes shocks financieros y económicos.

El objetivo principal de nuestra investigación es determinar la existencia del canal de préstamos bancarios en moneda nacional y extranjera durante el periodo que va desde el primer trimestre de 2010 hasta el primer trimestre de 2019. Para tal fin, se utilizó un modelo IS-LM con sector bancario. Dada la naturaleza de las series de tiempo, el análisis se basa en pruebas de causalidad a lo granger, en la aplicación del método de Johansen y en estimar modelos de corrección de errores tipo VECM.

De este modo, encontramos los siguientes resultados: (i) existe una relación de largo plazo entre los fondos de encaje en moneda nacional y el nivel de préstamos en soles, lo cual ante un incremento en un punto porcentual de los fondos de encaje en soles causan un aumento de los créditos en soles de 2,22\%; (ii) existe una relación de largo plazo entre los fondos de encaje en moneda nacional y el PBI real, así ante un incremento de $1 \%$ de los fondos de encaje en soles se produce un incremento del PBI real de 0,50\% en largo plazo; (iii) los fondos de encaje en moneda extranjera son buenos predictores de los créditos en dólares, de esta manera un incremento de $1 \%$ en los fondos de encaje en moneda extranjera causan un aumento de 4,89\% en los créditos en dólares; $y$, (iv) los fondos de encaje en dólares no son buenos predictores de la actividad económica. En concreto, se determina que el canal de préstamos bancarios en moneda nacional estaría operando durante el periodo de análisis. Sin embargo, no existe evidencia empírica sobre un canal de créditos en moneda extranjera.

Bajo estos resultados, la investigación está en línea con lo encontrado en otros países. Los autores Su, Gan y Hu (2010) demuestran la existencia del canal de préstamos bancarios en China, sin embargo el efecto de su política de encaje es menor que la peruana. Por otro lado, los autores Mello y Pisu (2009) determinan la existencia del canal de préstamos bancarios para la economía de Brasil. Así, su efecto de política de encaje afecta a los préstamos en $0.097 \%$ mientras que en Perú es de $2.22 \%$. 
Cabe señalar que el modelo teórico va de la mano con los resultados obtenidos para el canal de préstamos bancarios en moneda nacional, sin embargo, no se obtiene lo esperado en moneda extranjera.

La evidencia del canal de préstamos bancarios en moneda nacional sugiere que las políticas monetarias implementadas por el BCRP están siendo efectivas durante el periodo de análisis, es decir, la autoridad monetaria cuenta con instrumentos no convencionales, por ejemplo, la tasa de requerimiento de encaje en moneda nacional, que pueden afectar el volumen de préstamos en moneda nacional, lo que a su vez está impactando en la actividad económica.

Por otro lado, las medidas implementadas por el BCRP para desdolarizar la economía peruana, también, están siendo efectivas en frenar la expansión del crédito en moneda extranjera, empero no existe una relación con la actividad económica. Por lo tanto, no existe un canal de préstamos bancarios en moneda extranjera. Una posible explicación podría ser que los bancos cuentan con otras fuentes de financiamiento en moneda extranjera para paliar las medidas tomadas por el BCRP.

Finalmente, se recomienda continuar con la política no convencional ya que está teniendo los resultados esperados sobre el canal de préstamos bancarios en moneda nacional. Asimismo, se sugiere continuar con la implementación de la desdolarización de la economía peruana, sin embargo, al no demostrar el canal de préstamos en dicha moneda se recomienda realizar el proceso de una manera moderada.

\section{Referencias bibliográficas}

Armas, Adrián; Castillo, Paul y Vega, Marco (2014). Inflation targeting and quantitative tightening: Effects of Reserve Requirements in Peru. Working Paper, No. 003, Banco Central de Reserva del Perú. 31p. Recuperado de http:// www.bcrp.gob.pe/docs/Publicaciones/Documentos-de-Trabajo/2014/documento-de-trabajo-03-2014.pdf

Bernanke, Ben and Blinder, Alan (1988). Credit, Money, and Aggregate Demand. American Economic Review, Vol. 78, No. 2, may, p. 435-439. (DOI): 10.3386 / w2534

Bringas, Paul y Tuesta, Vicente (1998). El superávit de encaje y los mecanismos de transmisión de la política monetaria: una aproximación. Working Paper, 
Banco Central de Reserva del Perú, p. 33-50. Recuperado de http://www. bcrp.gob.pe/docs/Publicaciones/Revista-Estudios-Economicos/01/Estudios-Economicos-1-2.pdf

Bustamante, José; Cuba, Walter y Nivin, Rafael (2019). Determinants of Credit Growth and the Bank Lending Channel in Peru: A Loan Level Analysis. Working Paper, No. 007, Banco Central de Reserva del Perú. 30p. Recuperado de https://www.bis.org/publ/work803.pdf

Caglarirmak, Nilgun y Karahan, Pinar (2016). An analysis on the efficiency of bank lending channel in Turkey. Journal of Business, Economics and Finance, Vol. 5, No. 2, p. 206-217. DOI: 10.17261/Pressacademia.2016219262

Carrera, César (2011). El canal del crédito bancario en el Perú: Evidencia y mecanismo de transmisión. Revista Estudios Económicos, No. 22, diciembre, p. 63-82. Recuperado de http://www.bcrp.gob.pe/docs/Publicaciones/Revista-Estudios-Economicos/22/ree-22-carrera.pdf

Castillo, Paul; Montoro, Carlos y Tuesta, Vicente (2006). Hechos Estilizados de la Economía Peruana. Working Paper, No. 05, Banco Central de Reserva del Perú. 51p. Recuperado de http://www.bcrp.gob.pe/docs/Publicaciones/ Revista-Estudios-Economicos/14/Estudios-Economicos-14-2.pdf

Contreras, Alex; Gondo, Rocío; Oré, Erick y Pérez Fernando (2019). Assessing the impact de-dollarization measures in Peru. Working Paper, No. 005, Banco Central de Reserva del Perú. 30p. Recuperado de http://www.bcrp.gob.pe/ docs/Publicaciones/Documentos-de-Trabajo/2018/documento-de-trabajo-009-2018.pdf

Court, Eduardo y Williams, Erick (2011). Estadísticas y Econometría Financiera. Buenos Aires: Cengage Learning. 589p.

De Gregorio, José (2012). Macroeconomía. Teoría y Políticas. Chile: PearsonEducación. 769p. Recuperado de http://www.degregorio.cl/pdf/Macroeconomia.pdf

Ehrmann, Michael; Gambacorta, Jorge; Sevestre, Patrick and Worms, Andreas (2001). Financial Systems and The Role of Banks in Monetary Policy Transmission in The Euro Area. Working paper, No. 105, European Central Bank. 53p. Recuperado de https://www.ecb.europa.eu/pub/pdf/scpwps/ecbwp105.pdf

Granger, Cliver (1988). Some Recent Developments in a Concept of Causality. Journal of Econometrics, Vol. 39, p. 199-211. https://doi.org/10.1016/03044076(88)90045-0 
Johansen, Soren y Juselius, Katarina (1990). Maximum Likelihood Estimation and Inference on Cointegration with Applications to the Demand for Money. Oxford Bulletin of Economics and Statistics, Vol. 52, No. 2, may, p. 169-210. https://doi.org/10.1111/j.1468-0084.1990.mp52002003.x

Kassem, Mohamad and Awdeh, Ali (2018). Detecting The Monetary Policy Lending Chanel in Lebanon. The Journal of Developing, Vol. 52, No. 2, spring, p. 163-175. Recuperado de https://www.researchgate.net/publication/317231310_Detecting_The_Monetary_Policy_Lending_Channel_In_ Lebanon/link/5a19bbb20f7e9be37f9a5962/download

Lahura, Erick (2011). An Empirical Analysis of the Credit-Output Relationship: Evidence from Peru. Working Paper, No. 018, Banco Central de Reserva del Perú. 24p. Recuperado de http://www.bcrp.gob.pe/docs/Publicaciones/ Documentos-de-Trabajo/2011/Documento-de-Trabajo-18-2011.pdf

Lahura, Erick y Vargas, Paul (2013). La Relación Dinámica entre el Sistema Financiero y el nivel de actividad real en el Perú: 1965-2011. Working Paper, No. 019, Banco Central de Reserva del Perú. 30p. Recuperado de http:// www.bcrp.gob.pe/docs/Publicaciones/Documentos-de-Trabajo/2013/documento-de-trabajo-19-2013.pdf

Lahura, Erick y Vega, Hugo (2011). El impulso Crediticio y el PBI en el Perú: 19922009. Working Paper, No. 001, Banco Central de Reserva del Perú. 40p. Recuperado de http://www.bcrp.gob.pe/docs/Publicaciones/Documentos-deTrabajo/2011/Documento-de-Trabajo-01-2011.pdf

Locmelis, Karlis y Mititel, Daniel (2015). Impact of Russia is 2014-2015 Crisis on the Dynamic Linkages between the Stock Markets of Russia, the EU and U.S. Bachelor Thesis. Stockholm School of Economics, The Republic of Latvia, 101p. Recuperado de https://www.makroekonomika.lv/sites/default/ files/07_locmelismititel.pdf

Mello, Luis y Pisu, Mario (2009). The Bank Lending Channel of Monetary Transmission in Brazil: A VECM Approach. OECD Economics Department Working Papers, No. 71. 23p. https://doi.org/10.1016/j.qref.2009.09.006

Nzengue, Christian y Faycal, Ibrahim (2011). Bank capital and macroeconomic shocks: a principal components analysis and vector error correction model VECM. International Journal of Economics and Finance studies, Vol. 3, No. 2, p. 69-77. Recuperado de https://dergipark.org.tr/tr/download/articlefile/256676

Pérez, Fernando y Vega, Marco (2014). The Dynamic Effects of Interest Rates and Reserve Requirements. Working Paper, No. 018, Banco Central de Reserva 
del Perú. 32p. Recuperado de http://www.bcrp.gob.pe/docs/Publicaciones/ Documentos-de-Trabajo/2014/documento-de-trabajo-18-2014.pdf

Rossini, Renzo y Vega, Marco (2007). El mecanismo de transmisión de la política monetaria en un entorno de dolarización financiera: El caso del Perú entre 1996 y 2006. Working Paper, No. 017, Banco Central de Reserva del Perú. 31p. Recuperado de http://suscripciones.bcrp.gob.pe/docs/Publicaciones/ Revista-Estudios-Economicos/14/Estudios-Economicos-14-1.pdf

Sun, Shuzhang; Gan, Christopher y Hu, Baiding (2010). Bank lending channel in China is monetary policy transmission mechanism: a VECM approach. Investment Management and Financial Innovations, Vol. 7, No. 2, April, p. 5969. Recuperado de https://businessperspectives.org/images/pdf/applications/publishing/templates/article/assets/3249/imfi_en_2010_02_Sun.pdf

Vega, Marco y Chávez, Joselin (2017). Propagación de Choques de Encaje en el Sistema Bancario Peruano. Working Paper, No. 004, Banco Central de Reserva del Perú. 37p.Recuperado de http://www.bcrp.gob.pe/docs/Publicaciones/Documentos-de-Trabajo/2017/documento-de-trabajo-04-2017.pdf

Viladecut, Hugo y Cabello, Miguel (2014). El canal de crédito en el Perú: Una aproximación SVAR. Revista de Estudios Económicos, No. 27, junio, p. 51-66. Recuperado de http://www.bcrp.gob.pe/docs/Publicaciones/Revista-Estudios-Economicos/27/ree-27-viladegut-cabello.pdf

Yarasevika, Samara; Tongato, Alfianisa and Citra, Ayu (2015). Bank Lending Channel in Indonesia is Monetary Policy Transmission: A VECM approach. 5th International Conference, Singapore, september, p. 27-32. Recuperado de http:// www.worldresearchlibrary.org/up_proc/pdf/62-144229776627-32.pdf

\section{Notas al final}

1 Artículo de investigación científica que se origina por la necesidad de entender mejor el mecanismo de transmisión de política monetaria a través del canal de préstamos bancarios en el Perú. Esta investigación es resultado del proyecto de tesis: "La profundización financiera y la actividad económica: Un análisis empírico para el Perú (1992-2018)".

2 Economista, Universidad Nacional del Callao, Callao, Perú. Egresado de la maestría en economía con mención en finanzas, Universidad Nacional Mayor de San Marcos, Lima, Perú. Profesor e Investigador del Instituto de Formación Bancaria IFB Certus, Lima, Perú. Correo-e: pespinozai@certus.edu.pe.

3 La serie créditos representa el total de colocaciones del sector bancario al sector privado. Asimismo, los datos fueron tomados de la página web del Banco Central de Reserva del Perú.

4 Cabe señalar que la actividad económica se medirá a través del cambio porcentual de la serie en niveles del PBI real. 5 Se define como la evolución de la relación que existe entre el nivel de créditos del sector bancario y el PBI.

6 Los errores estimados son ruido blanco.

7 A pesar de esto, se estimó la ecuación de largo plazo resultando los coeficientes no significativos. 
